\title{
Numerical investigation of microstructure effect on mechanical properties of bi-continuous and particulate reinforced composite materials
}

\author{
Hailong Chen ${ }^{\mathrm{a}, 1}$, Lingyi Meng ${ }^{\mathrm{b}}$, Shaohua Chen ${ }^{\mathrm{a}}$, Yang Jiao ${ }^{\mathrm{a}}$, Yongming Liu ${ }^{\mathrm{a}, 2}$ \\ ${ }^{a}$ School for Engineering of Matter, Transport and Energy, Arizona State University, Tempe, AZ \\ 85287, US \\ ${ }^{b}$ School of Civil Engineering and Transportation, South China University of Technology, \\ Guangzhou 510641, China
}

\begin{abstract}
In this paper, numerical simulations are proposed to investigate mechanical properties of bicontinuous and particulate reinforced composite materials using a non-local voxel-based discrete computational model. Special focus of this article is the effect of 3D microstructure and its heterogeneity on elastic deformation and fracture behaviors. First, a review on model formulation is presented. Model parameters are derived in terms of material constants using the concept of energy equivalency. Interface representation and numerical homogenization scheme are discussed. Following this, numerical investigations on the effects of interface properties and inclusion characteristics, i.e. the volume fraction and material constants, on homogenized elastic constants and fracture behaviors of statistically isotropic bi-phase composites are performed. The effective elastic constants predicted by the proposed model agree well with analytical results. Fracture simulation demonstrates good capability of the

\footnotetext{
${ }^{1}$ Current Address: Fuels Modeling and Simulation Department, Idaho National Laboratory, Idaho Falls, ID 83402

${ }^{2}$ Corresponding author. Tel.: +1 4809656883

E-mail address: Yongming.Liu@asu.edu
} 
proposed model for the microstructure-sensitive failure analysis. Conclusions and future work are drawn based on the proposed study.

Keywords: Voxel-based analysis; Microstructure; Homogenization; Fracture; Composites

\section{Introduction}

Significant progresses in advanced imaging techniques, such as Electron Backscatter Diffraction (EBSD) [1] and X-ray tomographic Microscopy [2], have enabled the incorporation of material microstructural information into mechanical modeling of materials, as reported in Refs. [3], [4], [5], [6], [7], [8]. On one hand, when micrographic information is limited (e.g., only twodimensional images are available) and plane strain/stress assumptions are valid under particular loading conditions, two-dimensional microstructure-based analyses can be used and essential mechanical behaviors of such materials can be obtained from corresponding simulations [9], [10], [11]. On the other hand, since most materials possess intrinsic threedimensional heterogeneous microstructure in nature [12], such as polycrystalline materials and composite materials, a three-dimensional analysis is usually required in order to fully characterize and understand the physical properties of such materials [11].

Three-dimensional microstructures can be obtained by direct imaging techniques and numerical reconstructions. Advanced imaging techniques, such as X-ray tomographic microscopy [2] and multi-scale imaging [13], have been widely used to obtain threedimensional microstructure with high-resolution for a wide range of heterogeneous materials, such as metal matrix composites [14]. In the meantime, a variety of three-dimensional microstructure reconstruction methods from limited two-dimensional information have been developed, such as the stochastic reconstruction procedure [15], [16], [17], [18], [19], [20] and 
the multi-point reconstruction method [21], [22], [23], [24]. The reconstruction techniques greatly reduce the experimental cost for full 3D imaging and enhance the computational materials investigation.

In this paper, we investigate the microstructure effect on mechanical properties of stochastically reconstructed three-dimensional bi-continuous and particulate reinforced composite materials. A three-dimensional voxel-based computational tool is proposed for the microstructure sensitive mechanical analysis. The paper is organized as follows: First, a brief review of the model formulation is given. The model parameters are derived based on the energy equivalency and the theory of hyper-elasticity. The interface representation and homogenization scheme for three-dimensional simulation are discussed. Following this, numerical studies on elastic homogenization and quasi-static fracture of bi-phase composite materials are performed. Effects of interface elastic properties, inclusion shape, volume fraction and spatial distribution on the effective elastic properties and fracture behaviors are investigated. Finally, conclusions are drawn based on this study.

\section{Voxel-based computational tool: a review}

In voxel-based models, a given volume of material is represented by a three-dimensional regular grid (array of voxels on periodic lattice). A voxel is a volume element, located at the lattice site following certain packing, such as the simple cubic packing shown in Fig. 1(a). The voxels are connected with their neighbors via axial springs. The interaction between a typical voxel pair is nonlocal in the sense that it not only depends on the voxel pair itself, but also has contribution from all neighboring voxels. Theoretically, a typical voxel can interact with all its 


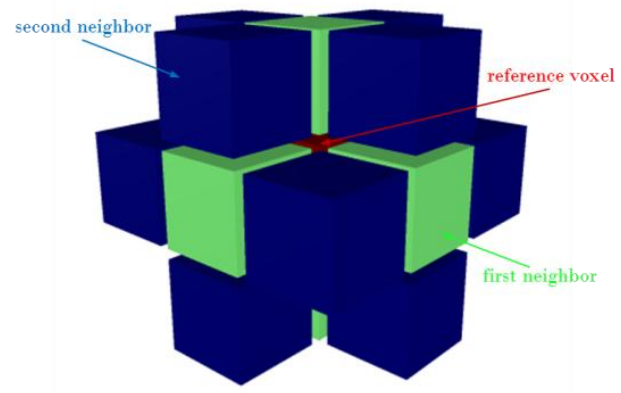
considered (see Fig. 1(b)).

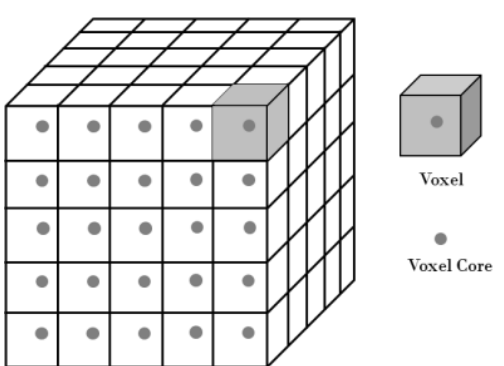

(a). Voxel representation of a bulk material

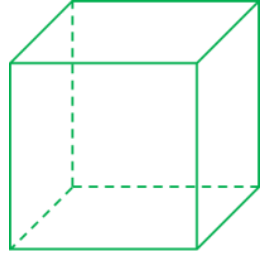

Unit cell 1:

Cube

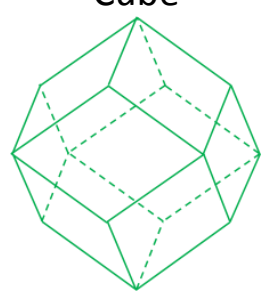

Unit cell 2:

rhombic dodecahedron

(c). Unit cells

Figure 1. The voxel system in the proposed model

\subsection{Model parameters derivation}

The derivation of model parameters in terms of material constants is based on the equivalency of energy [25], i.e., the potential energy of a voxel should be identical in both the continuum and discrete descriptions. In the discrete description, the potential energy is described based on the concept of the unit cell. A unit cell is a repeating non-overlapping unit identified from the geometric relationship between a reference voxel and its neighbors. For the two-neighbor case in this study, the unit cell for the first nearest neighbors is a cube and the unit cell for the second nearest neighbors is a rhombic dodecahedron, as shown in Fig. 1(c). For a given packing, the corresponding unit normal vectors of a typical voxel can be calculated. For the case of simple cubic packing with first and second nearest neighbors, the normal vectors are given in 
Table 1 . These unit normal vectors will be used to calculate the potential energy of a typical voxel.

Table 1. List of normal vectors of a typical voxel for the simple cubic packing

\begin{tabular}{lccc}
$n_{1}$ & $(1,0,0)$ & $n_{10}$ & $(-\sqrt{2} / 2,-\sqrt{2} / 2,0)$ \\
$n_{2}$ & $n_{11}$ & $(\sqrt{2} / 2,0, \sqrt{2} / 2)$ \\
$n_{3}$ & $n_{12}$ & $(0, \sqrt{2} / 2, \sqrt{2} / 2)$ \\
$n_{4}$ & $n_{13}$ & $(-\sqrt{2} / 2,0, \sqrt{2} / 2)$ \\
$n_{5}$ & $(-1,0,0)$ & $n_{14}$ & $(0,-\sqrt{2} / 2, \sqrt{2} / 2)$ \\
$n_{6}$ & $(0,-1,0)$ & $n_{15}$ & $(\sqrt{2} / 2,0,-\sqrt{2} / 2)$ \\
$n_{7}$ & $(0,0,1)$ & $n_{16}$ & $(0, \sqrt{2} / 2,-\sqrt{2} / 2)$ \\
$n_{8}$ & $(0,0,-1)$ & $n_{17}$ & $(-\sqrt{2} / 2,0,-\sqrt{2} / 2)$ \\
$n_{9}$ & $(\sqrt{2} / 2,-\sqrt{2} / 2,0)$ & $n_{18}$ & $(0,-\sqrt{2} / 2,-\sqrt{2} / 2)$ \\
\hline
\end{tabular}

The total potential energy of a reference voxel is the sum of the energies from these two unit cells, which can be written as

$$
U_{\text {voxel_i } i}=\sum_{k=1}^{2} U_{\text {cell_k }}^{i}
$$

And for each unit cell the energy has two parts

$$
U_{\text {cell_k }}^{i}=U_{\text {local_k }}^{i}+U_{\text {nonlocal_k }}^{i}
$$

where

$$
U_{\text {local_k }}^{i}=\frac{1}{2}\left(\alpha_{k}^{i} \sum_{j=1}^{N_{k}^{i}}\left(\delta l_{k j}^{i} \cdot \delta l_{k j}^{i}\right)\right)
$$

is the pair-wise local potential energy, and

$$
U_{\text {nonlocal_k }}^{i}=\frac{1}{2} t_{k}^{i}\left(\sum_{j=1}^{N_{k}^{i}} \delta I_{k j}^{i}\right) \cdot\left(\sum_{j=1}^{N_{k}^{i}} \delta I_{k j}^{i}\right)
$$

is the non-local multi-body potential energy of unit cell $k$ of voxel $i$. 
In Eqs. (3)-(4), $\alpha_{k}^{i}$ and $t_{k}^{i}$ are the pairwise and multi-body parameters for springs associated with unit cell $k$ of voxel $i$; $N_{k}^{i}$ is the total number of neighbors for unit cell $k$ of voxel $i$, and $\delta l_{k j}^{i}$ is the half length of spring elongation of spring $j$ of unit cell $k$ of voxel $i$.

Given the potential of each voxel, i.e., Eqs. (1)-(4), the interaction between a reference voxel and its neighbor $j$ can be calculated by differentiating the potential energy with respect to its length change as

$$
f_{k j}^{i}=-\frac{\partial U_{\text {voxel_i}}}{\partial\left(\delta I_{k j}^{i}\right)}=-\alpha_{k}^{i}\left(\delta I_{k j}^{i}\right)-t_{k}^{i}\left(\sum_{m=1}^{N_{k}^{i}} \delta I_{k m}^{i}\right)
$$

The effective spring stiffness can be obtained from Eq. (5) as

$$
\alpha_{k j}^{i}=\alpha_{k}^{i}+t_{k}^{i}\left(\sum_{m=1}^{N_{k}^{i}}\left(\frac{\delta l_{k m}^{i}}{\delta l_{k j}^{i}}\right)\right)
$$

From Eq.(6), it is clear that the effective spring stiffness for each spring in the proposed lattice spring model is non-local. This nonlocality removes the well-known restriction on materials' Poisson's ratio of classical lattice spring models [26].

If the spring elongations are related to components of strain tensor $\varepsilon$ at the continuum level, the potential of unit cell $k$ can be rewritten in terms of strain components and spring normal vectors given in Table 1 as

$$
U_{\text {cell_k }}^{i}=\frac{1}{2}\left(I_{k}\right)^{2}\left(\alpha_{k}^{i} \sum_{b=1}^{N_{k}^{i}}\left(n_{l}^{b} \varepsilon_{l J} n_{J}^{b} n_{K}^{b} \varepsilon_{K L} n_{L}^{b}\right)+t_{k}^{i}\left(\sum_{b=1}^{N_{k}^{i}} n_{l}^{b} \varepsilon_{I J} n_{J}^{b}\right)\left(\sum_{b=1}^{N_{k}^{i}} n_{K}^{b} \varepsilon_{K L} n_{L}^{b}\right)\right)
$$

where $I_{k}$ is the distance between the reference voxel $i$ with its $k$ th neighbor at the undeformed condition, i.e. the original spring length. $I_{1}$ equals to a voxel length and $I_{2}=\sqrt{2} I_{1}$. 
By conservation of the potential energy, the material stiffness tensor according to the theory of hyper-elasticity for a reference voxel can be obtained as

$$
\begin{aligned}
C_{\text {IJKL }}^{i} & =\frac{1}{V_{1}} \frac{\partial^{2}\left(U_{\text {cell_1 }}^{i}\right)}{\partial \varepsilon_{J J} \partial \varepsilon_{K L}}+\frac{V_{2}}{V_{1}}\left(\frac{1}{V_{2}} \frac{\partial^{2}\left(U_{c e l I_{2}}^{i}\right)}{\partial \varepsilon_{J J} \partial \varepsilon_{K L}}\right) \\
& =\frac{1}{8} \alpha_{1}^{i} \sum_{b=1}^{6} n_{l}^{b} n_{J}^{b} n_{K}^{b} n_{L}^{b}+\frac{1}{8} t_{1}^{i}\left(\sum_{b=1}^{6} n_{1}^{b} n_{J}^{b}\right)\left(\sum_{b=1}^{6} n_{K}^{b} n_{L}^{b}\right)+\frac{1}{4} \alpha_{2}^{i} \sum_{b=7}^{18} n_{l}^{b} n_{J}^{b} n_{K}^{b} n_{L}^{b}+\frac{1}{4} t_{2}^{i}\left(\sum_{b=7}^{18} n_{l}^{b} n_{J}^{b}\right)\left(\sum_{b=7}^{18} n_{K}^{b} n_{L}^{b}\right)
\end{aligned}
$$

where $V_{1}=l_{1}^{3}$ is the volume of the unit cell $1 . V_{2}=2 l_{1}^{3}$ is the volume of the unit cell 2.

In the proposed study, it is assumed that the nonlocal parameters for the two unit cells are identical for Hookean isotropic materials. Thus, the model parameters in terms of material's Young's modulus and Poisson's ratio can be uniquely solved as

$$
\begin{aligned}
\alpha_{1}^{i} & =\frac{l_{1} E}{1+v}, \quad \alpha_{2}^{i}=\frac{l_{1} E}{1+v} \\
t^{i} & =\frac{l_{1} E(4 v-1)}{18(1+v)(1-2 v)}
\end{aligned}
$$

where $E$ and $v$ are the Young's modulus and Poisson's ratio of a material.

The stress and strain tensors at each reference voxel center can also be calculated based on the potential energy as

$$
\begin{aligned}
\boldsymbol{\sigma}_{i} & =\frac{1}{V_{1}} \frac{\partial U_{\text {voxel_i}}}{\partial \boldsymbol{\varepsilon}_{i}} \\
& =\frac{1}{V_{i}} \sum_{n=1}^{2} \sum_{j=1}^{N_{n}^{i}} I_{n j}^{i} \frac{\partial U_{c e l l \_n}^{i}}{\partial \delta I_{n j}^{i}} \frac{\partial \boldsymbol{e}_{n j}^{i}}{\partial \boldsymbol{\varepsilon}_{i}} \\
& =\frac{1}{V_{1}} \sum_{n=1}^{2} \sum_{j=1}^{N_{n}^{i}} I_{n j}^{i}\left(\alpha_{n}^{i} \delta I_{n j}^{i}+T_{n}^{i} \sum_{k=1}^{N_{n}^{i}} \delta I_{n k}^{i}\right) \mathbf{n}_{n j}^{i}\left(\mathbf{n}_{n j}^{i}\right)^{T}
\end{aligned}
$$

and

$$
\boldsymbol{\varepsilon}_{i}=\boldsymbol{\sigma}_{i} \cdot \mathbf{S}_{i}
$$


where $\mathbf{S}_{i}$ is the compliance matrix of voxel $i$.

The above derivation of model parameters follows the same procedure as described in Ref. [9]. The only difference is that the final form of the potential energy and hence the material stiffness matrix is different. This is true for different packing systems as shown in Ref. [27].

\subsection{Interface representation}

Many studies have been devoted to analyze the interface effect on particulate composites using different interface models [28]. As discussed in Ref. [9], interface is intrinsic in discrete models and it is represented by springs straddling across different phases. By assigning different interface parameters for interface springs, interface effects can be effectively considered in these models. A schematic diagram illustrating the interface representation for 3D voxel-based modeling is shown in Fig. (2). Different color indicates different phases and springs.

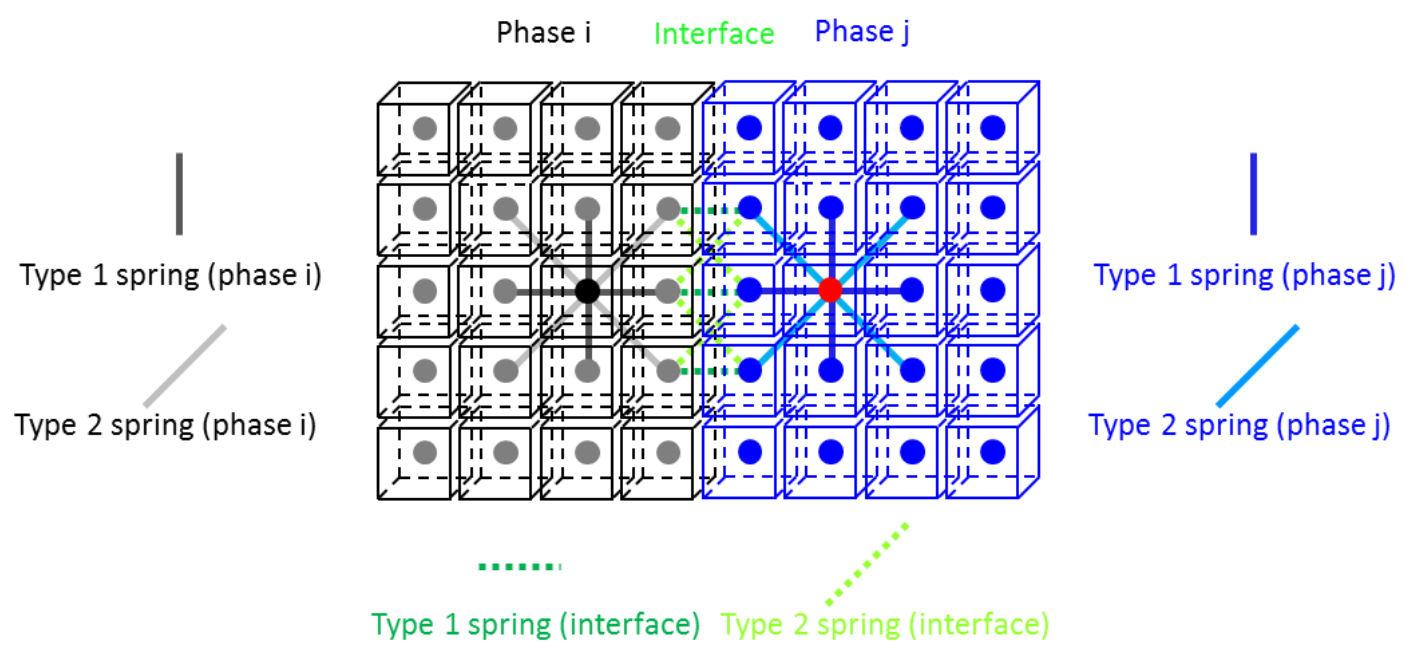

Figure 2. Interface representation in the proposed model

\subsection{Homogenization scheme}


Many analytical and numerical homogenization schemes are available in the literature, such as Hashin-Shtrikman bounds [29], third-order approximation method [30], and energy based numerical approaches [31]. In this section, the homogenization procedure presented in Refs. [9] and [31] for two-dimensional analysis is extended to three-dimensional cases.

The calculation of the effective elastic moduli is conducted in the following three steps:

(i) Apply uniform tri-axial extension to the investigated microstructure domain, corresponding to a strain state $\varepsilon_{11}=\varepsilon_{22}=\varepsilon_{33}=\varepsilon_{0}$, and calculate the total strain energy $U_{(1)}$ as a sum of energies of all voxels;

(ii) Apply uniform bi-axial extension on four faces and apply uniform uniaxial compression on the other two faces, which is corresponding to the strain state of $-\varepsilon_{11}=\varepsilon_{22}=\varepsilon_{33}=\varepsilon_{0}$. Following this, the total strain energy $U_{(2)}^{i}$ is calculated. This step is repeated three times by rotating the bi-axial strain state to other faces and the average calculated strain energy $U_{(2)}=\frac{1}{3} \sum_{i=1}^{3} U_{(2)}^{i}$ is chosen as the strain energy for this step;

In terms of the bulk modulus $K$ and shear modulus $G$, the strain energy of a three-dimensional homogeneous isotropic linear elastic continuum of volume $V$ is

$$
U=V\left(\frac{K}{2} \varepsilon_{i i} \varepsilon_{j j}+G\left(\varepsilon_{i j} \varepsilon_{i j}-\frac{1}{3} \varepsilon_{i i} \varepsilon_{i j}\right)\right)
$$

Thus, the bulk modulus and shear modulus can be obtained using the strain energy data as

$$
\begin{aligned}
& K=\frac{2}{9} \frac{U_{(1)}}{V \varepsilon_{0}^{2}} \\
& G=\frac{1}{24} \frac{9 U_{(2)}-U_{(1)}}{V \varepsilon_{0}^{2}}
\end{aligned}
$$


The Poisson's ratio in terms of the bulk and shear moduli is

$$
v=\frac{3 K-2 G}{2(3 K+G)}
$$

Thus, the model parameters can be derived in terms of the material constants using the concept of energy equivalency and the theory of hyper-elasticity. In next section, the proposed 3D voxel based model will be used in the microstructure-sensitive mechanical analysis of different composite systems.

\section{Numerical results}

The microstructure systems used in this study are generated using the stochastic reconstruction procedure (for bi-continuous composites) and the hard-particle Monte Carlo (MC) simulations [32] (for particulate composites). Typical systems generated using these methods are shown in Fig. 3, with the red color indicating the inclusion and the blue color representing the matrix. Simulations in this section will be based on these two digitized microstructures. The dimensions are assumed to be $0.01 \mathrm{~m}$ along each edges and the domain is discretized with a voxel density of $128 \times 128 \times 128$.

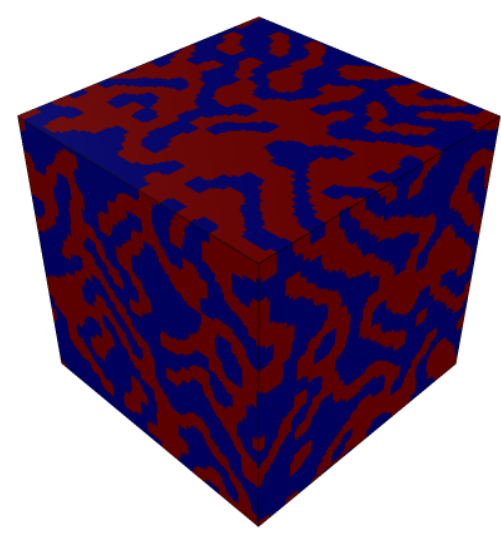

(a). Bi-continuous case

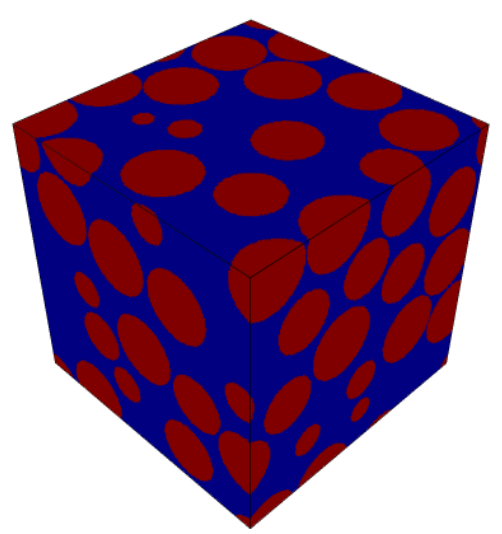

(b). Particulate case

Figure 3. Microstructures for different bi-phase composite systems used in this study (v.f. 50\%) 
The material properties for different phases are: Young's modulus $E_{m}=110 \mathrm{GPa}, v_{m}=0.34$ and $E_{i}=450 \mathrm{GPa}, v_{i}=0.17$, where $m$ indicates the matrix and $i$ for the inclusion. For the elastic homogenization problem, the implicit solution scheme developed in Ref. [33] is used, while the explicit solution scheme discussed in Ref. [26], [34] is used for quasi-static fracture problems.

\subsection{Elastic homogenization}

In this subsection, the proposed voxel-based discrete model is applied to estimate the effective elastic properties of aforementioned two composite systems. The procedure described in Section 2.3 is carried out for this purpose. We consider both the inclusion volume fraction effect and the interface effect on the effective elastic properties of these two composite systems. Two types of interface properties are used, i.e., interface 1 assumes the interface has the same properties as the inclusion while interface 2 assigns matrix properties to the interface. The obtained results are shown in Fig. 4 for bi-continuous composite system and Fig. 6 for particulate reinforced composite system. As can be seen, the predicted effective constants, bulk modulus and shear modulus, do not exactly fit with the Hashin-Shtrikman (H-S) bound [29] for all inclusion volume fractions, which is different from the observation from the twodimensional analysis [9]. The interface effect is more important for the three-dimensional analysis than the two-dimensional analysis in determining the effective material constants of composite systems. This is due to the fact that for a fixed inclusion volume fraction, the interface volume fraction is larger for the three-dimensional case than that for the twodimensional case. 

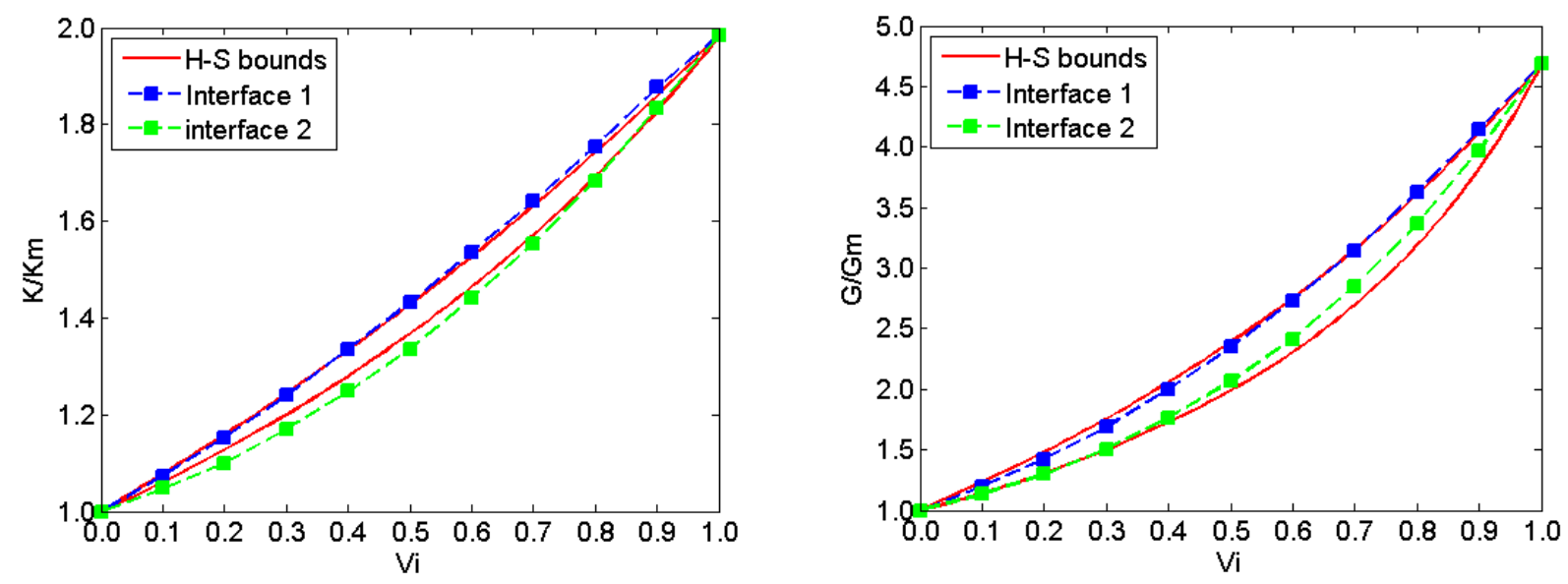

Figure 4. The effective elastic moduli for bi-continuous composite system
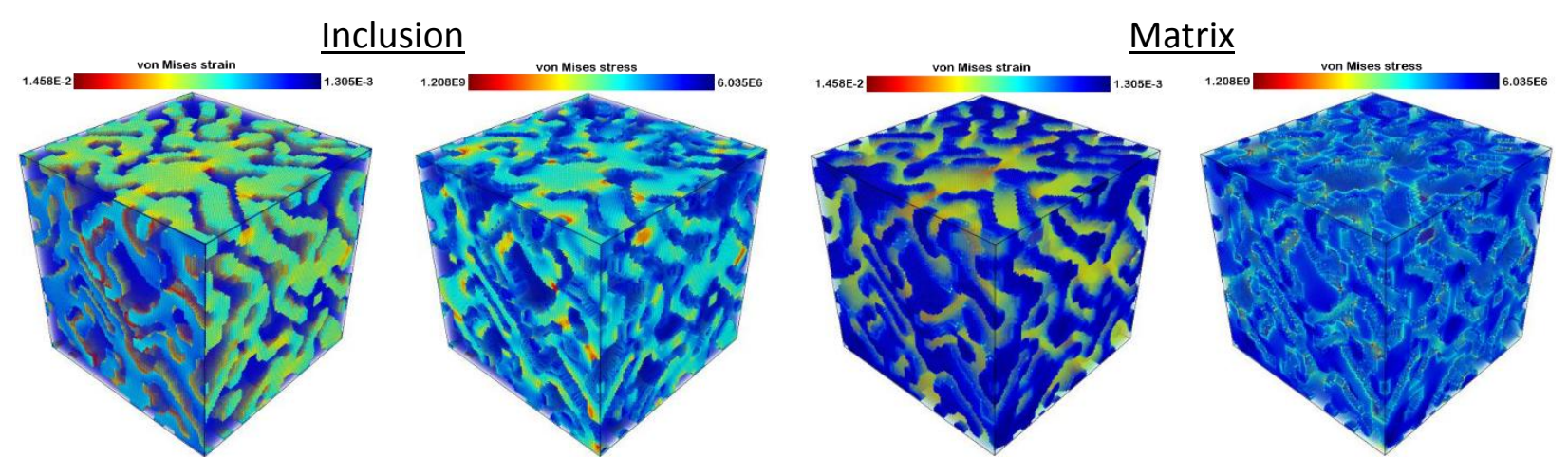

Figure 5. The von Mises stress and strain distributions for bi-continuous composite system

$$
(\text { vol\% }=50)
$$

The calculated von Mises strain and stress using the Eqs. (10) and (11) for the case of $50 \%$ inclusion volume fraction are shown in Fig. 5 and Fig. 7 for bi-continuous and particulate composite systems, respectively. As can be seen, the stress concentration occurs at the matrixinclusion interface. This is due to the discontinuity of material properties at the interface. 

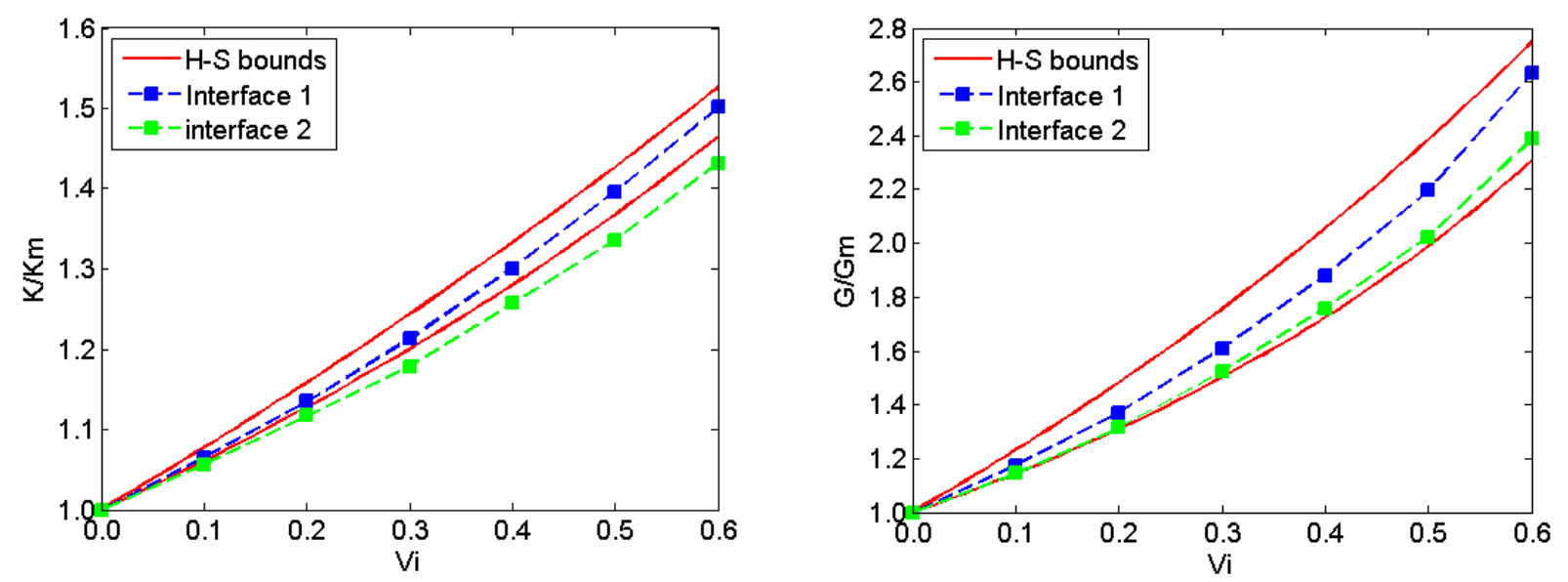

Figure 6. The effective elastic moduli for particulate composite system
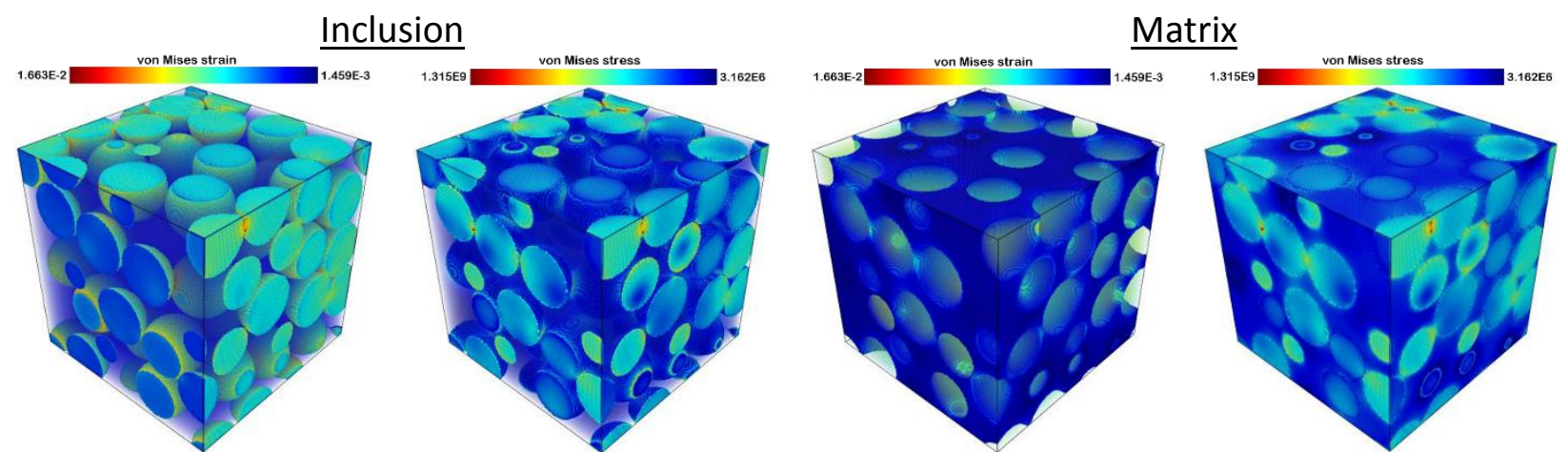

Figure 7. The von Mises stress and strain distributions for particulate composite system (vol\% = 50)

\subsection{Fracture modeling}

The fracture modeling of general solid material is a nontrivial problem in the literature. The complexity of the microstructure of reinforced composites adds additional difficulties. In this example, the proposed voxel-based model is applied to study the fracture behaviors, e.g., interfacial debonding, of particulate composites. This problem has been shown to be critical for the strength of particulate composite and has been investigated extensively, such as [35].

Three different particulate composites are considered in this example. All these systems have the same inclusion volume fraction and inclusion size, but with different inclusion distribution. Two descriptors are used to characterize the particulate distribution, i.e., the two-point 
correlation function $S 2(r)$ and the lineal-path function $L(r)$. The two-point correlation function S2(r) gives the probability of finding two points in the phases of interest [12], [36], [37]. The lineal-path function $L(r)$ gives the probability that a randomly chosen line segment of length $r$ entirely falls into the phase of interest [12], [38]. The corresponding $S 2(r)$ and $L(r)$ for the particulate inclusion phase are calculated and shown in Fig. 8. As can be seen, among the three different composites, the probabilities of finding two points in the inclusion phases are slightly different while the probabilities that a randomly chosen line segment entirely falls into the inclusion phase are the same.
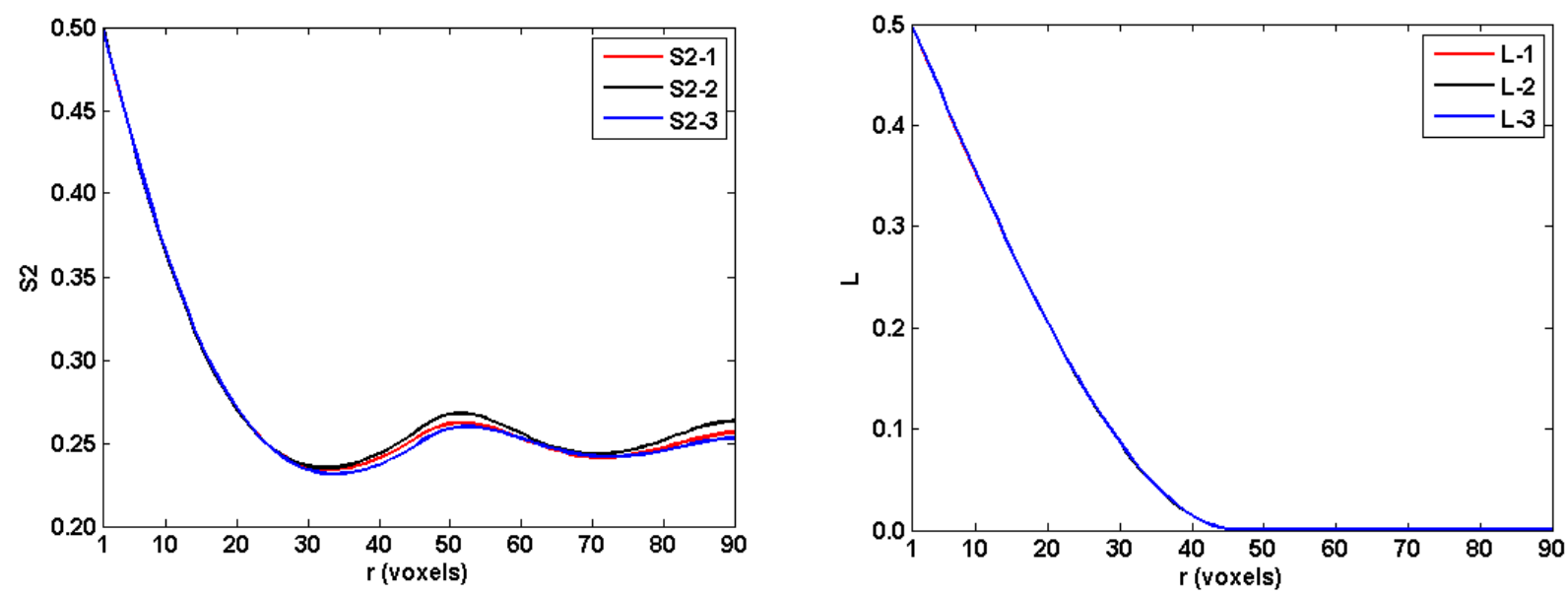

Figure 8. The $S 2$ and $L$ functions for three different particulate composite systems

For all three composites, the interfaces are assumed to have the properties of the matrix. Constant loading rates are applied on the top and bottom surfaces to simulate the uniaxial tension loading case. The critical elongation failure criterion is used and the critical values being $0.1 \%$ and $0.1 \% / \sqrt{2}$ for springs connecting the first and the second neighbors, respectively. Periodic boundary condition is applied on the lateral surfaces in a way similar to the Molecular Dynamic simulations (MD). 
The final crack surface for three different composites are shown in Fig. 9. The color indicates phases, i.e., the red one is the inclusion while the blue one is the matrix. The crack surfaces 1 to 3 correspond to the $\mathrm{S} 2$ and $\mathrm{L}$ curves in Fig. 8 . As can be seen, the major failure mechanism of the particulate reinforced composites is the interfacial debonding, which is consistent with the experimental observation [39].

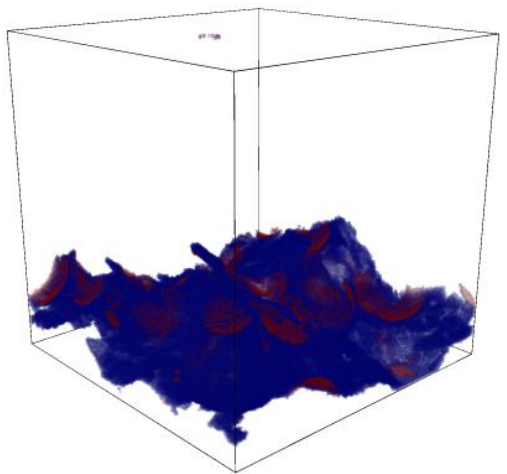

crack surface 1

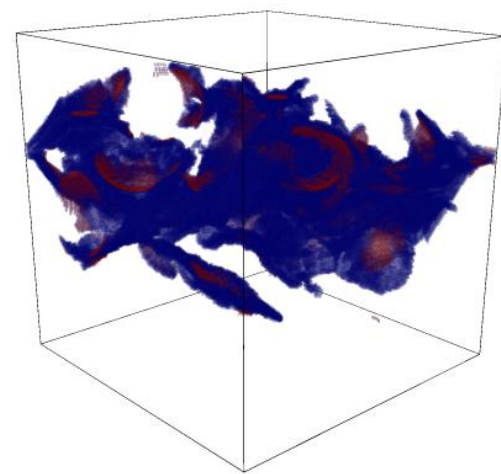

crack surface 2

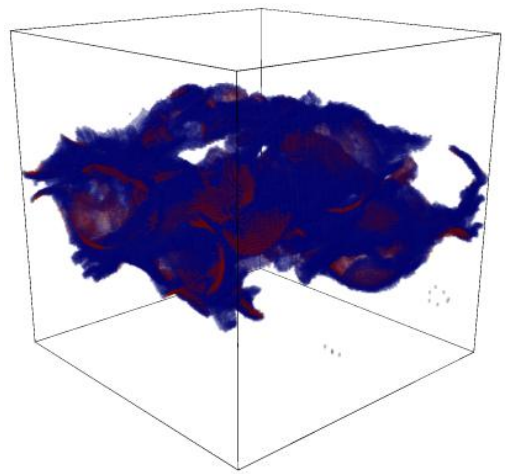

crack surface 3

Figure 9. The crack surfaces for three different particulate composites

Statistical information obtained from the three crack surfaces are as follows: For the inclusion phase, the ratios of voxels on the crack surface to the total number of inclusion voxels are $1.2 \%$, $1.3 \%$ and $1.6 \%$, respectively. For the matrix phase, the ratios are $9.6 \%, 10.5 \%$ and $12.7 \%$, respectively. The ratios of voxels on the crack surface to the total number of voxels are $5.4 \%$, $5.9 \%$ and $7.2 \%$, respectively.

The reaction forces on the top surfaces versus the applied strains are shown in Fig. 10. Microstructure 1 has the smallest peak reaction force and microstructure 3 has the largest peak reaction force. This is consistent with the statistical data from the crack surfaces show in Fig. 9. This is due to the larger energy required for larger crack surfaces. 


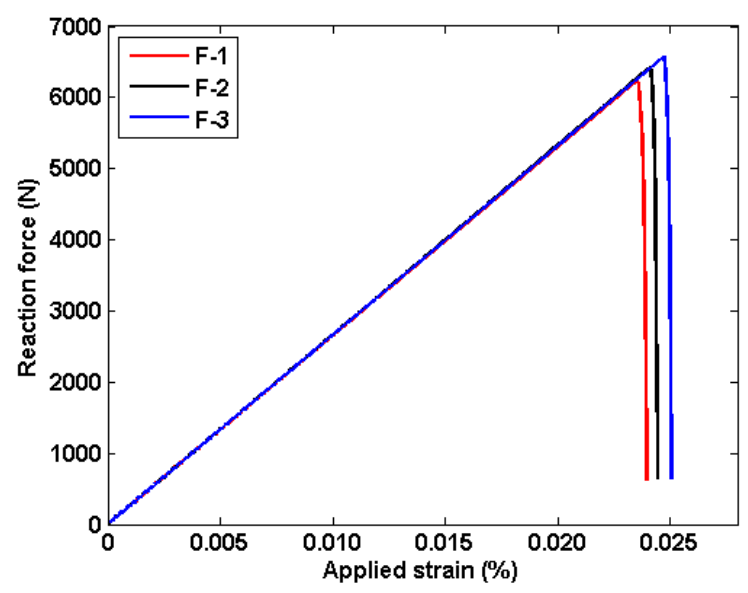

Figure 10. The reaction force on the top face versus applied strain

\section{Conclusions}

In this paper, a three-dimensional voxel-based numerical investigation of microstructure effect on mechanical properties of bi-continuous and particulate reinforced composite materials was presented. In the proposed computational model, both the first and second nearest neighbors of a voxel were considered. The local and nonlocal model parameters were derived using the concept of energy equivalency and the theory of hyper-elasticity. The interface was represented by springs straddling different phases. The fracture behaviors were modelled using a spring-based critical failure criterion.

The proposed voxel-based computational tool can be effectively applied to the microstructuresensitive mechanical analysis of composite materials. For elastic homogenization, the effective constants calculated using the proposed computational tool are consistent with other analytical predictions. The interfacial debonding failure mechanism of particulate reinforced composites is accurately captured.

In the current study, no material defects have been considered in any of the simulation. As has been reported in [40] and [41], evolution of defects, such as grain boundaries in crystalline 


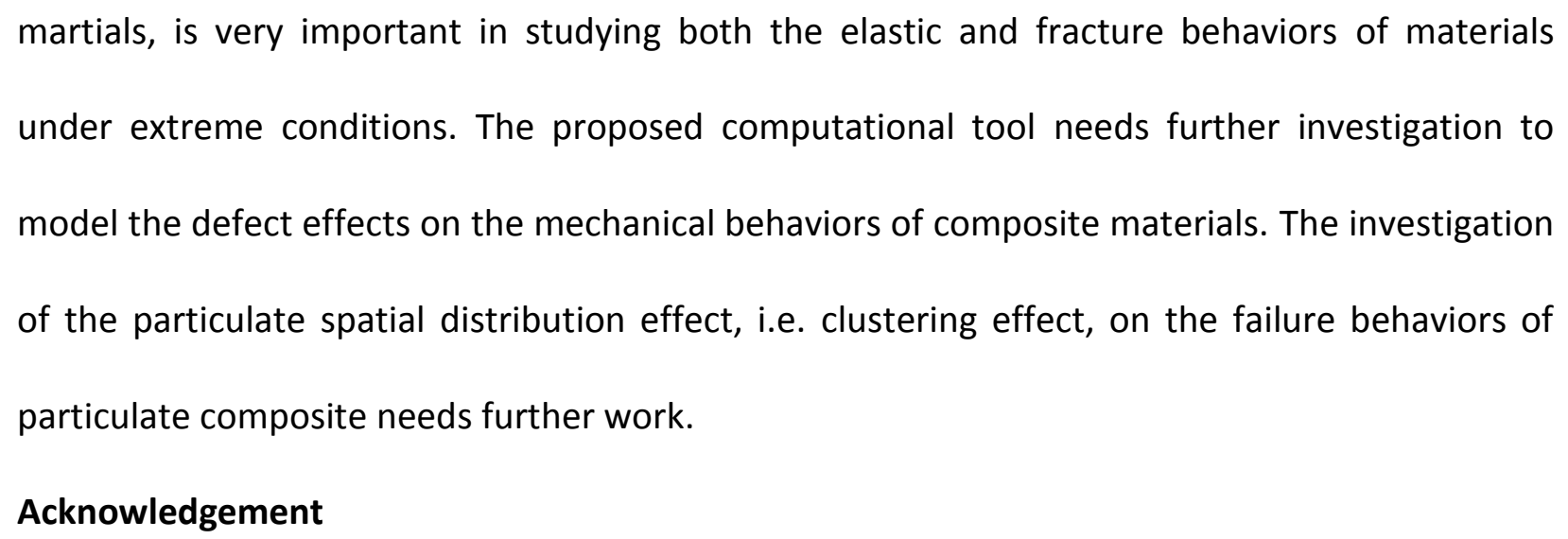

Acknowledgement

This work is partially supported by DARPA under grant No. N66001-14-1-4036. 


\section{References}

1. Randle, V., Applications of electron backscatter diffraction to materials science: status in 2009. Journal of Materials Science, 2009. 44(16): p. 4211-4218.

2. Kinney, J.H. and M.C. Nichols, X-Ray Tomographic Microscopy (XTM) Using Synchrotron Radiation. Annual Review of Materials Science, 1992. 22(1): p. 121-152.

3. Mishnaevsky Jr, L.L., Automatic voxel-based generation of 3D microstructural FE models and its application to the damage analysis of composites. Materials Science and Engineering: A, 2005. 407(1-2): p. 11-23.

4. Kim, J.H., M.-G. Lee, and R.H. Wagoner, A boundary smoothing algorithm for image-based modeling and its application to micromechanical analysis of multi-phase materials. Computational Materials Science, 2010. 47(3): p. 785-795.

5. Tarleton, E., M.N. Charalambides, and C. Leppard, Image-based modelling of binary composites. Computational Materials Science, 2012. 64: p. 183-186.

6. Alghamdi, A., P. Mummery, and M.A. Sheikh, Multi-scale 3D image-based modelling of a carbon/carbon composite. Modelling and Simulation in Materials Science and Engineering, 2013. 21(8): p. 085014.

7. Sun, B., X. Wang, and Z. Li, Meso-scale image-based modeling of reinforced concrete and adaptive multi-scale analyses on damage evolution in concrete structures. Computational Materials Science, 2015. 110: p. 39-53.

8. Zhang, J., et al., Crack initiation and fatigue life prediction on aluminum lug joints using statistical volume element-based multiscale modeling. Journal of Intelligent Material Systems and Structures, 2012.

9. Chen, H., et al., A novel discrete computational tool for microstructure-sensitive mechanical analysis of composite materials. Materials Science and Engineering: A, 2016. 659: p. 234-241.

10. Amsellem, O., et al., Two-dimensional (2D) and three-dimensional (3D) analyses of plasmasprayed alumina microstructures for finite-element simulation of Young's modulus. Journal of Materials Science, 2008. 43(12): p. 4091-4098.

11. Böhm, H.J. and W. Han, Comparisons between three-dimensional and two-dimensional multiparticle unit cell models for particle reinforced metal matrix composites. Modelling and Simulation in Materials Science and Engineering, 2001. 9(2): p. 47.

12. Torquato, S., Random heterogeneous media: microstructure and improved bounds on effective properties. Appl. Mech. Rev., 1991(44): p. 37-76.

13. Gerke, K.M., M.V. Karsanina, and D. Mallants, Universal Stochastic Multiscale Image Fusion: An Example Application for Shale Rock. Scientific Reports, 2015. 5: p. 15880.

14. Borbély, A., et al., Three-dimensional characterization of the microstructure of a metal-matrix composite by holotomography. Materials Science and Engineering: A, 2004. 367(1-2): p. 40-50.

15. Yeong, C.L.Y. and S. Torquato, Reconstructing random media. II. Three-dimensional media from two-dimensional cuts. Physical Review E, 1998. 58(1): p. 224-233.

16. $\mathrm{Xu}, \mathrm{H}$., et al., Descriptor-based methodology for statistical characterization and $3 D$ reconstruction of microstructural materials. Computational Materials Science, 2014. 85: p. 206216.

17. Chen, S., H. Li, and Y. Jiao, Dynamic reconstruction of heterogeneous materials and microstructure evolution. Physical Review E, 2015. 92(2): p. 023301.

18. Gerke, K.M. and M.V. Karsanina, Improving stochastic reconstructions by weighting correlation functions in an objective function. EPL, 2015. 111(5). 
19. Hasanabadi, A., et al., 3D microstructural reconstruction of heterogeneous materials from $2 D$ cross sections: A modified phase-recovery algorithm. Computational Materials Science, 2016. 111: p. 107-115.

20. Chen, S., et al., Stochastic Multi-Scale Reconstruction of 3D Microstructure Consisting of Polycrystalline Grains and Second-Phase Particles from 2D Micrographs. Metallurgical and Materials Transactions A, 2016. 47(3): p. 1440-1450.

21. Okabe, H. and M.J. Blunt, Pore space reconstruction using multiple-point statistics. Journal of Petroleum Science and Engineering, 2005. 46(1-2): p. 121-137.

22. Avery, P., C. Farhat, and G. Reese, Fast frequency sweep computations using a multi-point Padébased reconstruction method and an efficient iterative solver. International Journal for Numerical Methods in Engineering, 2007. 69(13): p. 2848-2875.

23. Hajizadeh, A., A. Safekordi, and F.A. Farhadpour, A multiple-point statistics algorithm for 3D pore space reconstruction from 2D images. Advances in Water Resources, 2011. 34(10): p. 1256-1267.

24. Staraselski, Y., et al., Reconstruction of the 3D representative volume element from the generalized two-point correlation function. Modelling and Simulation in Materials Science and Engineering, 2015. 23(1): p. 015007.

25. Chen, H., Y. Jiao, and Y. Liu, A Nonlocal Lattice Particle Model for Fracture Simulation of Anisotropic Materials. Composites Part B: Engineering, 2016. 90: p. 141-151.

26. Chen, H., E. Lin, and Y. Liu, A novel Volume-Compensated Particle method for 2D elasticity and plasticity analysis. International Journal of Solids and Structures, 2014. 51(9): p. 1819-1833.

27. Chen, H. and Y. Liu, A non-local 3D lattice particle framework for elastic solids. International Journal of Solids and Structures, 2016. 81: p. 411-420.

28. Xu, W.X. and H.S. Chen, Analytical and modeling investigations of volume fraction of interfacial layers around ellipsoidal aggregate particles in multiphase materials. Modelling and Simulation in Materials Science and Engineering, 2013. 21(1): p. 015005.

29. Hashin, Z. and S. Shtrikman, A variational approach to the theory of the elastic behaviour of multiphase materials. Journal of the Mechanics and Physics of Solids, 1963. 11(2): p. 127-140.

30. Torquato, S., Effective stiffness tensor of composite media: II. Application to isotropic dispersions. Journal of the Mechanics and Physics of Solids, 1998(46): p. 1411-1440.

31. Chen, H., Y. Jiao, and Y. Liu, Investigating the Microstructural Effect on Elastic and Fracture Behavior of Polycrystals Using a Nonlocal Lattice Particle Model. Materials Science and Engineering: A, 2015. 631: p. 173-180.

32. Torquato, S. and Y. Jiao, Dense packings of polyhedra: Platonic and Archimedean solids. Phys. Rev. E, 2009. 80(4): p. 041104.

33. Lin, E., H. Chen, and Y. Liu, Finite Element Implementation of A Non-local Particle Method for Elasticity and Fracture Analysis. Finite Elements in Analysis and Design, 2015. 93(0): p. 1-11.

34. Chen, H., et al., A Generalized 2D Non-local Lattice Spring Model for Fracture Simulation. Computational Mechanics, 2014. 54(6): p. 1541-1558.

35. Jiang, Y. and K. Tohgo, An incremental damage theory for micropolar composites taking account of progressive debonding and particle size effect. Computational Materials Science, 2011. 50(12): p. 3358-3364.

36. Jiao, Y., F.H. Stillinger, and S. Torquato, Modeling heterogeneous materials via two-point correlation functions: Basic principles. Physical Review E, 2007. 76(3): p. 031110.

37. Jiao, Y., F.H. Stillinger, and S. Torquato, Modeling heterogeneous materials via two-point correlation functions. II. Algorithmic details and applications. Physical Review E, 2008. 77(3): p. 031135.

38. Lu, B. and S. Torquato, Lineal-path function for random heterogeneous materials. Physical Review A, 1992. 45(2): p. 922-929. 
39. Bindumadhavan, P.N., H.K. Wah, and O. Prabhakar, Assessment of particle-matrix debonding in particulate metal matrix composites using ultrasonic velocity measurements. Materials Science and Engineering: A, 2002. 323(1-2): p. 42-51.

40. Chen, Z., et al., Dislocation climb strengthening in systems with immobile obstacles: Threedimensional level-set simulation study. Physical Review B, 2010. 81(5): p. 054104.

41. Lim, A.T., et al., Stress-driven migration of simple low-angle mixed grain boundaries. Acta Materialia, 2012. 60(3): p. 1395-1407. 


\section{Numerical investigation of microstructure effect on mechanical properties of bi-continuous}

\section{and particulate reinforced composite materials}

Hailong Chen , Lingyi Meng, Shaohua Chen, Yang Jiao, Yongming Liu

\section{Graphical Abstract}
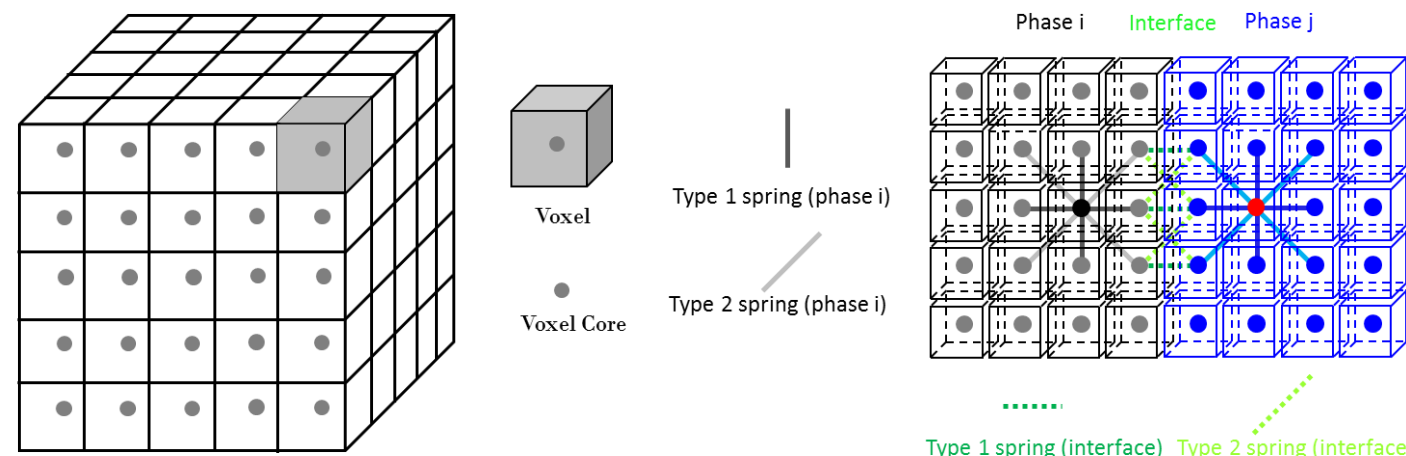

Type 1 spring (phase j)

Type 1 spring (phase i)

Type 2 spring (phase i)

Type 1 spring (interface) Type 2 spring (interface)

Voxel representation of materials

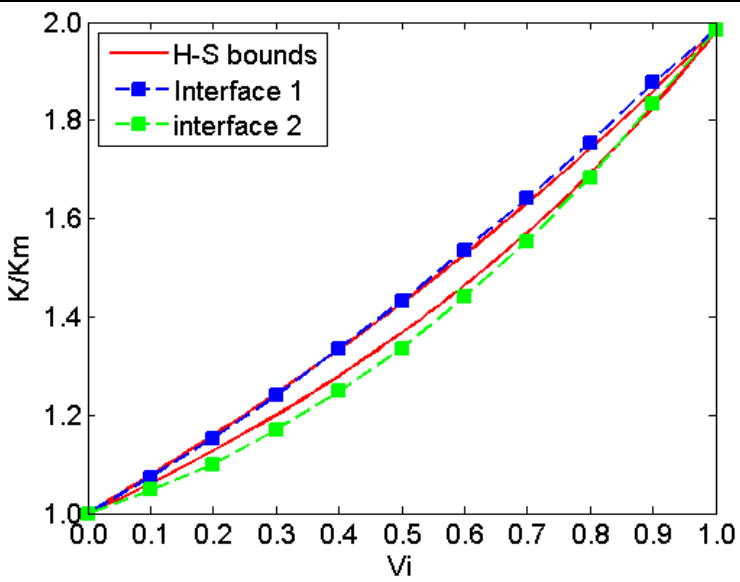

Voxels are connected by springs whose properties are uniquely derived from material constants

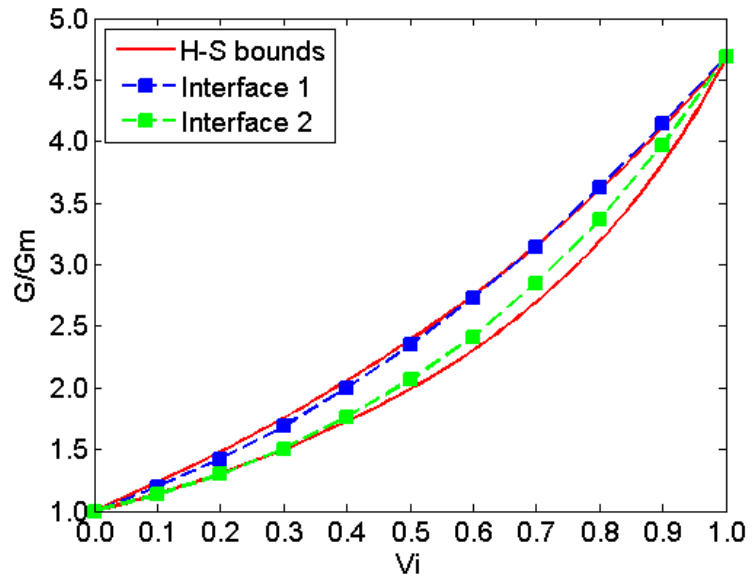

The interface property effect on homogenized elastic moduli of bi-continuous composite 